\title{
Basic Operations on Preordered Coherent Spaces
}

\author{
Klaus E. Grue \\ DIKU \\ University of Copenhagen \\ Universitetsparken 1 \\ DK-2100 Copenhagen \\ Denmark
}

\author{
Artur Korniłowicz ${ }^{1}$ \\ Institute of Computer Science \\ University of Białystok \\ Sosnowa 64, 15-887 Białystok \\ Poland
}

Summary. This Mizar paper presents the definition of a "Preordered Coherent Space" (PCS). Furthermore, the paper defines a number of operations on PCS's and states and proves a number of elementary lemmas about these operations. PCS's have many useful properties which could qualify them for mathematical study in their own right. PCS's were invented, however, to construct Scott domains, to solve domain equations, and to construct models of various versions of lambda calculus.

For more on PCS's, see [11]. The present Mizar paper defines the operations on PCS's used in Chapter 8 of [3].

MML identifier: PCS_0, version: 7.8.05 4.87.985

The articles [16], [20], [7], [17], [15], [21], [4], [6], [22], [23], [14], [1], [13], [5], [18], [9], [19], [12], [8], [2], and [10] provide the notation and terminology for this paper.

\section{Preliminaries}

Let $R_{1}, R_{2}$ be sets and let $R$ be a relation between $R_{1}$ and $R_{2}$. Then field $R$ is a subset of $R_{1} \cup R_{2}$.

\footnotetext{
${ }^{1}$ The author visited the Department of Computer Science, University of Copenhagen, while writing the article.
} 
Let $R_{1}, R_{2}, S_{1}, S_{2}$ be sets, let $R$ be a relation between $R_{1}$ and $R_{2}$, and let $S$ be a relation between $S_{1}$ and $S_{2}$. Then $R \cup S$ is a relation between $R_{1} \cup S_{1}$ and $R_{2} \cup S_{2}$.

Let $R_{1}, S_{1}$ be sets, let $R$ be a total binary relation on $R_{1}$, and let $S$ be a total binary relation on $S_{1}$. Note that $R \cup S$ is total.

Let $R_{1}, S_{1}$ be sets, let $R$ be a reflexive binary relation on $R_{1}$, and let $S$ be a reflexive binary relation on $S_{1}$. Observe that $R \cup S$ is reflexive.

Let $R_{1}, S_{1}$ be sets, let $R$ be a symmetric binary relation on $R_{1}$, and let $S$ be a symmetric binary relation on $S_{1}$. Observe that $R \cup S$ is symmetric.

One can prove the following proposition

(1) Let $R_{1}, S_{1}$ be sets, $R$ be a transitive binary relation on $R_{1}$, and $S$ be a transitive binary relation on $S_{1}$. If $R_{1}$ misses $S_{1}$, then $R \cup S$ is transitive.

Let $A$ be an empty set and let $B$ be a set. One can check that $\emptyset_{A, B}$ is total.

Let $I$ be a non empty set and let $C$ be a 1 -sorted yielding many sorted set indexed by $I$. Then the support of $C$ can be characterized by the condition:

(Def. 1) For every element $i$ of $I$ holds (the support of $C)(i)=$ the carrier of $C(i)$.

Let $R_{1}, R_{2}, S_{1}, S_{2}$ be sets, let $R$ be a relation between $R_{1}$ and $R_{2}$, and let $S$ be a relation between $S_{1}$ and $S_{2}$. The functor [ $R, S^{\wedge}$ ] yields a relation between : $R_{1}, S_{1}$ : and : $R_{2}, S_{2}:$ and is defined by the condition (Def. 2).

(Def. 2) Let $x, y$ be sets. Then $\langle x, y\rangle \in\left[{ }^{\wedge} R, S^{\wedge}\right]$ if and only if there exist sets $r_{1}$, $s_{1}, r_{2}, s_{2}$ such that $x=\left\langle r_{1}, s_{1}\right\rangle$ and $y=\left\langle r_{2}, s_{2}\right\rangle$ and $r_{1} \in R_{1}$ and $s_{1} \in S_{1}$ and $r_{2} \in R_{2}$ and $s_{2} \in S_{2}$ and $\left\langle r_{1}, r_{2}\right\rangle \in R$ or $\left\langle s_{1}, s_{2}\right\rangle \in S$.

Let $R_{1}, R_{2}, S_{1}, S_{2}$ be non empty sets, let $R$ be a relation between $R_{1}$ and $R_{2}$, and let $S$ be a relation between $S_{1}$ and $S_{2}$. Then $\left[{ }^{\wedge} R, S^{\wedge}\right]$ can be characterized by the condition:

(Def. 3) Let $r_{1}$ be an element of $R_{1}, r_{2}$ be an element of $R_{2}, s_{1}$ be an element of $S_{1}$, and $s_{2}$ be an element of $S_{2}$. Then $\left\langle\left\langle r_{1}, s_{1}\right\rangle,\left\langle r_{2}, s_{2}\right\rangle\right\rangle \in\left[{ }^{\wedge} R, S^{\wedge}\right]$ if and only if $\left\langle r_{1}, r_{2}\right\rangle \in R$ or $\left\langle s_{1}, s_{2}\right\rangle \in S$.

Let $R_{1}, S_{1}$ be sets, let $R$ be a total binary relation on $R_{1}$, and let $S$ be a total binary relation on $S_{1}$. Note that [^ $\left.R, S^{\wedge}\right]$ is total.

Let $R_{1}, S_{1}$ be sets, let $R$ be a reflexive binary relation on $R_{1}$, and let $S$ be a reflexive binary relation on $S_{1}$. One can check that $\left[{ }^{\wedge} R, S^{\wedge}\right]$ is reflexive.

Let $R_{1}, S_{1}$ be sets, let $R$ be a binary relation on $R_{1}$, and let $S$ be a total reflexive binary relation on $S_{1}$. Observe that $\left[{ }^{\wedge} R, S^{\wedge}\right]$ is reflexive.

Let $R_{1}, S_{1}$ be sets, let $R$ be a total reflexive binary relation on $R_{1}$, and let $S$ be a binary relation on $S_{1}$. Observe that $\left[{ }^{\wedge} R, S^{\wedge}\right]$ is reflexive.

Let $R_{1}, S_{1}$ be sets, let $R$ be a symmetric binary relation on $R_{1}$, and let $S$ be a symmetric binary relation on $S_{1}$. Note that $\left[{ }^{\wedge} R, S^{\wedge}\right]$ is symmetric. 


\section{Relational Structures}

Let us observe that every relational structure which is empty is also total. if:

Let $R$ be a binary relation. We say that $R$ is transitive-yielding if and only

(Def. 4) For every relational structure $S$ such that $S \in \operatorname{rng} R$ holds $S$ is transitive.

Let us note that every binary relation which is poset-yielding is also transitive-yielding.

Let us mention that there exists a function which is poset-yielding.

Let $I$ be a set. Observe that there exists a many sorted set indexed by $I$ which is poset-yielding.

Let $I$ be a set and let $C$ be a relational structure yielding many sorted set indexed by $I$. The functor pcs-InternalRels $C$ yields a many sorted set indexed by $I$ and is defined by the condition (Def. 5).

(Def. 5) Let $i$ be a set. Suppose $i \in I$. Then there exists a relational structure $P$ such that $P=C(i)$ and (pcs-InternalRels $C)(i)=$ the internal relation of $P$.

Let $I$ be a non empty set and let $C$ be a relational structure yielding many sorted set indexed by $I$. Then pcs-InternalRels $C$ can be characterized by the condition:

(Def. 6) For every element $i$ of $I$ holds (pcs-InternalRels $C)(i)=$ the internal relation of $C(i)$.

Let $I$ be a set and let $C$ be a relational structure yielding many sorted set indexed by $I$. One can check that pcs-InternalRels $C$ is binary relation yielding.

Let $I$ be a non empty set, let $C$ be a transitive-yielding relational structure yielding many sorted set indexed by $I$, and let $i$ be an element of $I$. Note that $C(i)$ is transitive.

\section{Tolerance Structures}

We introduce alternative relational structures which are extensions of 1sorted structure and are systems

$\langle$ a carrier, an alternative relation 〉,

where the carrier is a set and the alternative relation is a binary relation on the carrier.

Let $P$ be an alternative relational structure and let $p, q$ be elements of $P$. The predicate $p \sim q$ is defined by:

(Def. 7) $\langle p, q\rangle \in$ the alternative relation of $P$.

Let $P$ be an alternative relational structure. We say that $P$ is $\beta$-total if and only if: 
(Def. 8) The alternative relation of $P$ is total.

We say that $P$ is $\beta$-reflexive if and only if:

(Def. 9) The alternative relation of $P$ is reflexive in the carrier of $P$.

We say that $P$ is $\beta$-irreflexive if and only if:

(Def. 10) The alternative relation of $P$ is irreflexive in the carrier of $P$.

We say that $P$ is $\beta$-symmetric if and only if:

(Def. 11) The alternative relation of $P$ is symmetric in the carrier of $P$.

The alternative relational structure emptyTolStr is defined as follows:

(Def. 12) emptyTolStr $=\left\langle\emptyset, \emptyset_{\emptyset, \emptyset}\right\rangle$.

One can check that emptyTolStr is empty and strict.

The following proposition is true

(2) Let $P$ be an alternative relational structure. If $P$ is empty, then the alternative relational structure of $P=$ emptyTolStr .

One can check that every alternative relational structure which is $\beta$-reflexive is also $\beta$-total.

Let us note that every alternative relational structure which is empty is also $\beta$-reflexive, $\beta$-irreflexive, and $\beta$-symmetric.

Let us note that there exists an alternative relational structure which is empty.

Let $P$ be a $\beta$-total alternative relational structure. Observe that the alternative relation of $P$ is total.

Let $P$ be a $\beta$-reflexive alternative relational structure. One can check that the alternative relation of $P$ is reflexive.

Let $P$ be a $\beta$-irreflexive alternative relational structure. One can verify that the alternative relation of $P$ is irreflexive.

Let $P$ be a $\beta$-symmetric alternative relational structure. One can verify that the alternative relation of $P$ is symmetric.

Let $L$ be a $\beta$-total alternative relational structure. Note that the alternative relational structure of $L$ is $\beta$-total.

Let $P$ be a $\beta$-symmetric alternative relational structure and let $p, q$ be elements of $P$. Let us note that the predicate $p \sim q$ is symmetric.

Let $D$ be a set. Note that $\left\langle D, \nabla_{D}\right\rangle$ is $\beta$-reflexive and $\beta$-symmetric.

Let $D$ be a set. Note that $\left\langle D, \emptyset_{D, D}\right\rangle$ is $\beta$-irreflexive and $\beta$-symmetric.

Let us note that there exists an alternative relational structure which is strict, non empty, $\beta$-reflexive, and $\beta$-symmetric.

One can check that there exists an alternative relational structure which is strict, non empty, $\beta$-irreflexive, and $\beta$-symmetric.

Let $R$ be a binary relation. We say that $R$ is alternative relational structure yielding if and only if: 
(Def. 13) For every set $P$ such that $P \in \operatorname{rng} R$ holds $P$ is an alternative relational structure.

Let $f$ be a function. Let us observe that $f$ is alternative relational structure yielding if and only if:

(Def. 14) For every set $x$ such that $x \in \operatorname{dom} f$ holds $f(x)$ is an alternative relational structure.

Let $I$ be a set and let $f$ be a many sorted set indexed by $I$. Let us observe that $f$ is alternative relational structure yielding if and only if:

(Def. 15) For every set $x$ such that $x \in I$ holds $f(x)$ is an alternative relational structure.

Let $R$ be a binary relation. We say that $R$ is $\beta$-reflexive yielding if and only if:

(Def. 16) For every alternative relational structure $S$ such that $S \in \operatorname{rng} R$ holds $S$ is $\beta$-reflexive.

We say that $R$ is $\beta$-irreflexive yielding if and only if:

(Def. 17) For every alternative relational structure $S$ such that $S \in \operatorname{rng} R$ holds $S$ is $\beta$-irreflexive.

We say that $R$ is $\beta$-symmetric yielding if and only if:

(Def. 18) For every alternative relational structure $S$ such that $S \in \operatorname{rng} R$ holds $S$ is $\beta$-symmetric.

One can check that every binary relation which is empty is also $\beta$-reflexive yielding, $\beta$-irreflexive yielding, and $\beta$-symmetric yielding.

Let $I$ be a set and let $P$ be an alternative relational structure. Note that $I \longmapsto P$ is alternative relational structure yielding.

Let $I$ be a set and let $P$ be a $\beta$-reflexive alternative relational structure. Observe that $I \longmapsto P$ is $\beta$-reflexive yielding.

Let $I$ be a set and let $P$ be a $\beta$-irreflexive alternative relational structure. One can check that $I \longmapsto P$ is $\beta$-irreflexive yielding.

Let $I$ be a set and let $P$ be a $\beta$-symmetric alternative relational structure. One can verify that $I \longmapsto P$ is $\beta$-symmetric yielding.

Let us observe that every function which is alternative relational structure yielding is also 1-sorted yielding.

Let $I$ be a set. Observe that there exists a many sorted set indexed by $I$ which is $\beta$-reflexive yielding, $\beta$-symmetric yielding, and alternative relational structure yielding.

Let $I$ be a set. Note that there exists a many sorted set indexed by $I$ which is $\beta$-irreflexive yielding, $\beta$-symmetric yielding, and alternative relational structure yielding.

Let $I$ be a set. Observe that there exists a many sorted set indexed by $I$ which is alternative relational structure yielding. 
Let $I$ be a non empty set, let $C$ be an alternative relational structure yielding many sorted set indexed by $I$, and let $i$ be an element of $I$. Then $C(i)$ is an alternative relational structure.

Let $I$ be a set and let $C$ be an alternative relational structure yielding many sorted set indexed by $I$. The functor pcs-ToleranceRels $C$ yields a many sorted set indexed by $I$ and is defined by the condition (Def. 19).

(Def. 19) Let $i$ be a set. Suppose $i \in I$. Then there exists an alternative relational structure $P$ such that $P=C(i)$ and (pcs-ToleranceRels $C)(i)=$ the alternative relation of $P$.

Let $I$ be a non empty set and let $C$ be an alternative relational structure yielding many sorted set indexed by $I$. Then pcs-ToleranceRels $C$ can be characterized by the condition:

(Def. 20) For every element $i$ of $I$ holds (pcs-ToleranceRels $C)(i)=$ the alternative relation of $C(i)$.

Let $I$ be a set and let $C$ be an alternative relational structure yielding many sorted set indexed by $I$. Note that pcs-ToleranceRels $C$ is binary relation yielding.

Let $I$ be a non empty set, let $C$ be a $\beta$-reflexive yielding alternative relational structure yielding many sorted set indexed by $I$, and let $i$ be an element of $I$. One can verify that $C(i)$ is $\beta$-reflexive.

Let $I$ be a non empty set, let $C$ be a $\beta$-irreflexive yielding alternative relational structure yielding many sorted set indexed by $I$, and let $i$ be an element of $I$. Note that $C(i)$ is $\beta$-irreflexive.

Let $I$ be a non empty set, let $C$ be a $\beta$-symmetric yielding alternative relational structure yielding many sorted set indexed by $I$, and let $i$ be an element of $I$. Observe that $C(i)$ is $\beta$-symmetric.

The following propositions are true:

(3) Let $P, Q$ be alternative relational structures. Suppose that

(i) the alternative relational structure of $P=$ the alternative relational structure of $Q$, and

(ii) $P$ is $\beta$-reflexive.

Then $Q$ is $\beta$-reflexive.

(4) Let $P, Q$ be alternative relational structures. Suppose that

(i) the alternative relational structure of $P=$ the alternative relational structure of $Q$, and

(ii) $\quad P$ is $\beta$-irreflexive.

Then $Q$ is $\beta$-irreflexive.

(5) Let $P, Q$ be alternative relational structures. Suppose that

(i) the alternative relational structure of $P=$ the alternative relational structure of $Q$, and

(ii) $\quad P$ is $\beta$-symmetric. 
Then $Q$ is $\beta$-symmetric.

Let $P, Q$ be alternative relational structures. The functor $\left[{ }^{\wedge} P, Q^{\wedge}\right]$ yields an alternative relational structure and is defined by the condition (Def. 21).

(Def. 21) $\quad\left[{ }^{\wedge} P, Q^{\wedge}\right]=\left\langle[\right.$ : the carrier of $P$, the carrier of $Q:],\left[{ }^{\wedge}\right.$ the alternative relation of $P$, the alternative relation of $\left.\left.Q^{\wedge}\right]\right\rangle$.

Let $P, Q$ be alternative relational structures, let $p$ be an element of $P$, and let $q$ be an element of $Q$. We introduce [^ $\left.{ }^{\wedge}, q^{\wedge}\right]$ as a synonym of $\langle p, q\rangle$.

Let $P, Q$ be non empty alternative relational structures, let $p$ be an element of $P$, and let $q$ be an element of $Q$. Then $\left[{ }^{\wedge} p, q^{\wedge}\right]$ is an element of $\left[{ }^{\wedge} P, Q^{\wedge}\right]$.

Let $P, Q$ be alternative relational structures and let $p$ be an element of $\left[{ }^{\wedge} P, Q^{\wedge}\right]$. We introduce $p^{\wedge} 1$ as a synonym of $p_{\mathbf{1}}$. We introduce $p^{\prime} 2$ as a synonym of $p_{\mathbf{2}}$.

Let $P, Q$ be non empty alternative relational structures and let $p$ be an element of $\left[{ }^{\wedge} P, Q^{\wedge}\right]$. Then $p^{\wedge} 1$ is an element of $P$. Then $p^{\prime} 2$ is an element of $Q$.

We now state two propositions:

(6) Let $S_{1}, S_{2}$ be non empty alternative relational structures, $a, c$ be elements of $S_{1}$, and $b, d$ be elements of $S_{2}$. Then [ $\left.{ }^{\wedge} a, b^{\wedge}\right] \sim\left[{ }^{\wedge} c, d^{\wedge}\right]$ if and only if $a \sim c$ or $b \sim d$.

(7) Let $S_{1}, S_{2}$ be non empty alternative relational structures and $x, y$ be elements of $\left[{ }^{\wedge} S_{1}, S_{2}^{\wedge}\right]$. Then $x \sim y$ if and only if one of the following conditions is satisfied:

(i) $x^{\prime} 1 \sim y^{\prime} 1$, or

(ii) $\quad x^{\prime} 2 \sim y^{\prime} 2$.

Let $P$ be an alternative relational structure and let $Q$ be a $\beta$-reflexive alternative relational structure. Note that $\left[{ }^{\wedge} P, Q^{\wedge}\right]$ is $\beta$-reflexive.

Let $P$ be a $\beta$-reflexive alternative relational structure and let $Q$ be an alternative relational structure. Observe that $\left[{ }^{\wedge} P, Q^{\wedge}\right]$ is $\beta$-reflexive.

Let $P, Q$ be $\beta$-symmetric alternative relational structures. One can check that $\left[{ }^{\wedge} P, Q^{\wedge}\right]$ is $\beta$-symmetric.

\section{PCS'S}

We introduce pcs structures which are extensions of relational structure and alternative relational structure and are systems

$\langle$ a carrier, an internal relation, an alternative relation $\rangle$, where the carrier is a set, the internal relation is a binary relation on the carrier, and the alternative relation is a binary relation on the carrier.

Let $P$ be a pcs structure. We say that $P$ is compatible if and only if:

(Def. 22) For all elements $p, p^{\prime}, q, q^{\prime}$ of $P$ such that $p \sim q$ and $p^{\prime} \leq p$ and $q^{\prime} \leq q$ holds $p^{\prime} \sim q^{\prime}$. 
Let $P$ be a pcs structure. We say that $P$ is pcs-like if and only if:

(Def. 23) $P$ is reflexive, transitive, $\beta$-reflexive, $\beta$-symmetric, and compatible.

We say that $P$ is anti-pcs-like if and only if:

(Def. 24) $P$ is reflexive, transitive, $\beta$-irreflexive, $\beta$-symmetric, and compatible.

One can verify the following observations:

* every pcs structure which is pcs-like is also reflexive, transitive, $\beta$ reflexive, $\beta$-symmetric, and compatible,

* every pcs structure which is reflexive, transitive, $\beta$-reflexive, $\beta$ symmetric, and compatible is also pcs-like,

* every pcs structure which is anti-pcs-like is also reflexive, transitive, $\beta$ irreflexive, $\beta$-symmetric, and compatible, and

* every pcs structure which is reflexive, transitive, $\beta$-irreflexive, $\beta$ symmetric, and compatible is also anti-pcs-like.

Let $D$ be a set. The functor TotalPCS $D$ yields a pcs structure and is defined as follows:

(Def. 25) TotalPCS $D=\left\langle D, \nabla_{D}, \nabla_{D}\right\rangle$.

Let $D$ be a set. Observe that TotalPCS $D$ is strict.

Let $D$ be a non empty set. One can verify that TotalPCS $D$ is non empty.

Let $D$ be a set. One can check that TotalPCS $D$ is reflexive, transitive, $\beta$-reflexive, and $\beta$-symmetric.

Let $D$ be a set. Note that TotalPCS $D$ is pcs-like.

Let $D$ be a set. One can verify that $\left\langle D, \nabla_{D}, \emptyset_{D, D}\right\rangle$ is anti-pcs-like.

One can verify that there exists a pcs structure which is strict, non empty, and pcs-like and there exists a pcs structure which is strict, non empty, and anti-pcs-like.

A pcs is a pcs-like pcs structure. An anti-pcs is an anti-pcs-like pcs structure.

The pcs structure EmptyPCS is defined by:

(Def. 26) $\quad$ EmptyPCS = TotalPCS 0.

Let us mention that EmptyPCS is strict, empty, and pcs-like.

Let $p$ be a set. The functor SingletonPCS $p$ yielding a pcs structure is defined by:

(Def. 27) SingletonPCS $p=$ TotalPCS $\{p\}$.

Let $p$ be a set. Observe that SingletonPCS $p$ is strict, non empty, and pcslike.

Let $R$ be a binary relation. We say that $R$ is pcs structure yielding if and only if:

(Def. 28) For every set $P$ such that $P \in \operatorname{rng} R$ holds $P$ is a pcs structure.

We say that $R$ is pcs-yielding if and only if:

(Def. 29) For every set $P$ such that $P \in \operatorname{rng} R$ holds $P$ is a pcs. 
Let $f$ be a function. Let us observe that $f$ is pcs structure yielding if and only if:

(Def. 30) For every set $x$ such that $x \in \operatorname{dom} f$ holds $f(x)$ is a pcs structure.

Let us observe that $f$ is pcs-yielding if and only if:

(Def. 31) For every set $x$ such that $x \in \operatorname{dom} f$ holds $f(x)$ is a pcs.

Let $I$ be a set and let $f$ be a many sorted set indexed by $I$. Let us observe that $f$ is pcs structure yielding if and only if:

(Def. 32) For every set $x$ such that $x \in I$ holds $f(x)$ is a pcs structure.

Let us observe that $f$ is pcs-yielding if and only if:

(Def. 33) For every set $x$ such that $x \in I$ holds $f(x)$ is a pcs.

One can verify the following observations:

* every binary relation which is pcs structure yielding is also alternative relational structure yielding and relational structure yielding,

* every binary relation which is pcs-yielding is also pcs structure yielding, and

* every binary relation which is pcs-yielding is also reflexive-yielding, transitive-yielding, $\beta$-reflexive yielding, and $\beta$-symmetric yielding.

Let $I$ be a set and let $P$ be a pcs. Note that $I \longmapsto P$ is pcs-yielding.

Let $I$ be a set. Observe that there exists a many sorted set indexed by $I$ which is pcs-yielding.

Let $I$ be a non empty set, let $C$ be a pcs structure yielding many sorted set indexed by $I$, and let $i$ be an element of $I$. Then $C(i)$ is a pcs structure.

Let $I$ be a non empty set, let $C$ be a pcs-yielding many sorted set indexed by $I$, and let $i$ be an element of $I$. Then $C(i)$ is a pcs.

Let $P, Q$ be pcs structures. The predicate $P \subseteq Q$ is defined by the conditions (Def. 34).

(Def. 34)(i) The carrier of $P \subseteq$ the carrier of $Q$,

(ii) the internal relation of $P \subseteq$ the internal relation of $Q$, and

(iii) the alternative relation of $P \subseteq$ the alternative relation of $Q$.

Let us note that the predicate $P \subseteq Q$ is reflexive.

Next we state two propositions:

(8) Let $P, Q$ be relational structures, $p, q$ be elements of $P$, and $p_{1}, q_{1}$ be elements of $Q$. Suppose the internal relation of $P \subseteq$ the internal relation of $Q$ and $p=p_{1}$ and $q=q_{1}$ and $p \leq q$. Then $p_{1} \leq q_{1}$.

(9) Let $P, Q$ be pcs structures, $p, q$ be elements of $P$, and $p_{1}, q_{1}$ be elements of $Q$. Suppose the alternative relation of $P \subseteq$ the alternative relation of $Q$ and $p=p_{1}$ and $q=q_{1}$ and $p \sim q$. Then $p_{1} \sim q_{1}$.

Let $C$ be a binary relation. We say that $C$ is chain-like if and only if: 
(Def. 35) For all pcs structures $P, Q$ such that $P \in \operatorname{rng} C$ and $Q \in \operatorname{rng} C$ holds $P \subseteq Q$ or $Q \subseteq P$.

Let $I$ be a set and let $P$ be a pcs structure. Observe that $I \longmapsto P$ is chain-like.

Let us note that there exists a function which is chain-like and pcs-yielding.

Let $I$ be a set. Note that there exists a many sorted set indexed by $I$ which is chain-like and pcs-yielding.

Let $I$ be a set. A pcs-chain of $I$ is a chain-like pcs-yielding many sorted set indexed by $I$.

Let $I$ be a set and let $C$ be a pcs structure yielding many sorted set indexed by $I$. The functor $\bigcup C$ yielding a strict pcs structure is defined by the conditions (Def. 36).

(Def. 36)(i) The carrier of $\bigcup C=\bigcup$ (the support of $C$ ),

(ii) the internal relation of $\bigcup C=\bigcup$ pcs-InternalRels $C$, and

(iii) the alternative relation of $\bigcup C=\bigcup$ pcs-ToleranceRels $C$.

We now state four propositions:

(10) Let $I$ be a set, $C$ be a pcs structure yielding many sorted set indexed by $I$, and $p, q$ be elements of $\bigcup C$. Then $p \leq q$ if and only if there exists a set $i$ and there exists a pcs structure $P$ and there exist elements $p^{\prime}, q^{\prime}$ of $P$ such that $i \in I$ and $P=C(i)$ and $p^{\prime}=p$ and $q^{\prime}=q$ and $p^{\prime} \leq q^{\prime}$.

(11) Let $I$ be a non empty set, $C$ be a pcs structure yielding many sorted set indexed by $I$, and $p, q$ be elements of $\bigcup C$. Then $p \leq q$ if and only if there exists an element $i$ of $I$ and there exist elements $p^{\prime}, q^{\prime}$ of $C(i)$ such that $p^{\prime}=p$ and $q^{\prime}=q$ and $p^{\prime} \leq q^{\prime}$.

(12) Let $I$ be a set, $C$ be a pcs structure yielding many sorted set indexed by $I$, and $p, q$ be elements of $\bigcup C$. Then $p \sim q$ if and only if there exists a set $i$ and there exists a pcs structure $P$ and there exist elements $p^{\prime}, q^{\prime}$ of $P$ such that $i \in I$ and $P=C(i)$ and $p^{\prime}=p$ and $q^{\prime}=q$ and $p^{\prime} \sim q^{\prime}$.

(13) Let $I$ be a non empty set, $C$ be a pcs structure yielding many sorted set indexed by $I$, and $p, q$ be elements of $\bigcup C$. Then $p \sim q$ if and only if there exists an element $i$ of $I$ and there exist elements $p^{\prime}, q^{\prime}$ of $C(i)$ such that $p^{\prime}=p$ and $q^{\prime}=q$ and $p^{\prime} \sim q^{\prime}$.

Let $I$ be a set and let $C$ be a reflexive-yielding pcs structure yielding many sorted set indexed by $I$. Observe that $\bigcup C$ is reflexive.

Let $I$ be a set and let $C$ be a $\beta$-reflexive yielding pcs structure yielding many sorted set indexed by $I$. Observe that $\bigcup C$ is $\beta$-reflexive.

Let $I$ be a set and let $C$ be a $\beta$-symmetric yielding pcs structure yielding many sorted set indexed by $I$. Note that $\bigcup C$ is $\beta$-symmetric.

Let $I$ be a set and let $C$ be a pcs-chain of $I$. One can check that $\bigcup C$ is transitive and compatible. 
Let $p, q$ be sets. The functor $\operatorname{MSSet}(p, q)$ yielding a many sorted set indexed by $\{0,1\}$ is defined by:

(Def. 37) $\operatorname{MSSet}(p, q)=[0 \longmapsto p, 1 \longmapsto q]$.

Let $P, Q$ be 1-sorted structures. One can check that $\operatorname{MSSet}(P, Q)$ is 1-sorted yielding.

Let $P, Q$ be relational structures. Observe that $\operatorname{MSSet}(P, Q)$ is relational structure yielding.

Let $P, Q$ be alternative relational structures. Observe that $\operatorname{MSSet}(P, Q)$ is alternative relational structure yielding.

Let $P, Q$ be pcs structures. Note that $\operatorname{MSSet}(P, Q)$ is pcs structure yielding.

Let $P, Q$ be reflexive pcs structures. Observe that $\operatorname{MSSet}(P, Q)$ is reflexiveyielding.

Let $P, Q$ be transitive pcs structures. One can check that $\operatorname{MSSet}(P, Q)$ is transitive-yielding.

Let $P, Q$ be $\beta$-reflexive pcs structures. Note that $\operatorname{MSSet}(P, Q)$ is $\beta$-reflexive yielding.

Let $P, Q$ be $\beta$-symmetric pcs structures. Observe that $\operatorname{MSSet}(P, Q)$ is $\beta$ symmetric yielding.

Let $P, Q$ be pcs's. Observe that $\operatorname{MSSet}(P, Q)$ is pcs-yielding.

Let $P, Q$ be pcs structures. The functor $P \oplus Q$ yielding a pcs structure is defined by:

(Def. 38) $P \oplus Q=\bigcup \operatorname{MSSet}(P, Q)$.

One can prove the following four propositions:

(14) Let $P, Q$ be pcs structures. Then

(i) the carrier of $P \oplus Q=($ the carrier of $P) \cup($ the carrier of $Q$ ),

(ii) the internal relation of $P \oplus Q=$ (the internal relation of $P$ ) $\cup$ (the internal relation of $Q$ ), and

(iii) the alternative relation of $P \oplus Q=($ the alternative relation of $P) \cup($ the alternative relation of $Q$ ).

(15) Let $P, Q$ be pcs structures. Then $P \oplus Q=\langle($ the carrier of $P) \cup$ (the carrier of $Q$ ), (the internal relation of $P) \cup($ the internal relation of $Q$ ), (the alternative relation of $P) \cup($ the alternative relation of $Q)\rangle$.

(16) Let $P, Q$ be pcs structures and $p, q$ be elements of $P \oplus Q$. Then $p \leq q$ if and only if one of the following conditions is satisfied:

(i) there exist elements $p^{\prime}, q^{\prime}$ of $P$ such that $p^{\prime}=p$ and $q^{\prime}=q$ and $p^{\prime} \leq q^{\prime}$, or

(ii) there exist elements $p^{\prime}, q^{\prime}$ of $Q$ such that $p^{\prime}=p$ and $q^{\prime}=q$ and $p^{\prime} \leq q^{\prime}$.

(17) Let $P, Q$ be pcs structures and $p, q$ be elements of $P \oplus Q$. Then $p \sim q$ if and only if one of the following conditions is satisfied:

(i) there exist elements $p^{\prime}, q^{\prime}$ of $P$ such that $p^{\prime}=p$ and $q^{\prime}=q$ and $p^{\prime} \sim q^{\prime}$, or 
(ii) there exist elements $p^{\prime}, q^{\prime}$ of $Q$ such that $p^{\prime}=p$ and $q^{\prime}=q$ and $p^{\prime} \sim q^{\prime}$.

Let $P, Q$ be reflexive pcs structures. Observe that $P \oplus Q$ is reflexive.

Let $P, Q$ be $\beta$-reflexive pcs structures. One can verify that $P \oplus Q$ is $\beta$ reflexive.

Let $P, Q$ be $\beta$-symmetric pcs structures. Observe that $P \oplus Q$ is $\beta$-symmetric.

The following three propositions are true:

(18) For all pcs's $P, Q$ such that $P$ misses $Q$ holds the internal relation of $P \oplus Q$ is transitive.

(19) For all pcs's $P, Q$ such that $P$ misses $Q$ holds $P \oplus Q$ is compatible.

(20) For all pcs's $P, Q$ such that $P$ misses $Q$ holds $P \oplus Q$ is a pcs.

Let $P$ be a pcs structure and let $a$ be a set. The functor $P_{a}$ yields a strict pcs structure and is defined by the conditions (Def. 39).

(Def. 39)(i) The carrier of $P_{a}=\{a\} \cup$ the carrier of $P$,

(ii) the internal relation of $P_{a}=:\{a\}$, the carrier of $\left.P_{a}:\right\}$ the internal relation of $P$, and

(iii) the alternative relation of $P_{a}=\left[:\{a\}\right.$, the carrier of $P_{a}: \cup[$ : the carrier of $P_{a},\{a\}: \cup$ the alternative relation of $P$.

Let $P$ be a pcs structure and let $a$ be a set. Observe that $P_{a}$ is non empty. The following propositions are true:

(21) Let $P$ be a pcs structure and $a$ be a set. Then

(i) the carrier of $P \subseteq$ the carrier of $P_{a}$,

(ii) the internal relation of $P \subseteq$ the internal relation of $P_{a}$, and

(iii) the alternative relation of $P \subseteq$ the alternative relation of $P_{a}$.

(22) For every pcs structure $P$ and for every set $a$ and for all elements $p, q$ of $P_{a}$ such that $p=a$ holds $p \leq q$.

(23) Let $P$ be a pcs structure, $a$ be a set, $p, q$ be elements of $P$, and $p_{1}, q_{1}$ be elements of $P_{a}$. If $p=p_{1}$ and $q=q_{1}$ and $p \leq q$, then $p_{1} \leq q_{1}$.

(24) Let $P$ be a pcs structure, $a$ be a set, $p$ be an element of $P$, and $p_{1}, q_{1}$ be elements of $P_{a}$. Suppose $p=p_{1}$ and $p \neq a$ and $p_{1} \leq q_{1}$ and $a \notin$ the carrier of $P$. Then $q_{1} \in$ the carrier of $P$ and $q_{1} \neq a$.

(25) Let $P$ be a pcs structure, $a$ be a set, and $p$ be an element of $P_{a}$. If $p \neq a$, then $p \in$ the carrier of $P$.

(26) Let $P$ be a pcs structure, $a$ be a set, $p, q$ be elements of $P$, and $p_{1}, q_{1}$ be elements of $P_{a}$. If $p=p_{1}$ and $q=q_{1}$ and $p \neq a$ and $p_{1} \leq q_{1}$, then $p \leq q$.

(27) For every pcs structure $P$ and for every set $a$ and for all elements $p, q$ of $P_{a}$ such that $p=a$ holds $p \sim q$ and $q \sim p$.

(28) Let $P$ be a pcs structure, $a$ be a set, $p, q$ be elements of $P$, and $p_{1}, q_{1}$ be elements of $P_{a}$. If $p=p_{1}$ and $q=q_{1}$ and $p \sim q$, then $p_{1} \sim q_{1}$. 
(29) Let $P$ be a pcs structure, $a$ be a set, $p, q$ be elements of $P$, and $p_{1}, q_{1}$ be elements of $P_{a}$. If $p=p_{1}$ and $q=q_{1}$ and $p \neq a$ and $q \neq a$ and $p_{1} \sim q_{1}$, then $p \sim q$.

Let $P$ be a reflexive pcs structure and let $a$ be a set. Observe that $P_{a}$ is reflexive.

The following proposition is true

(30) For every transitive pcs structure $P$ and for every set $a$ such that $a \notin$ the carrier of $P$ holds $P_{a}$ is transitive.

Let $P$ be a $\beta$-reflexive pcs structure and let $a$ be a set. One can verify that $P_{a}$ is $\beta$-reflexive.

Let $P$ be a $\beta$-symmetric pcs structure and let $a$ be a set. One can check that $P_{a}$ is $\beta$-symmetric.

Next we state two propositions:

(31) For every compatible pcs structure $P$ and for every set $a$ such that $a \notin$ the carrier of $P$ holds $P_{a}$ is compatible.

(32) For every pcs $P$ and for every set $a$ such that $a \notin$ the carrier of $P$ holds $P_{a}$ is a pcs.

Let $P$ be a pcs structure. The functor $\uparrow P$ yields a strict pcs structure and is defined by the conditions (Def. 40).

(Def. 40)(i) The carrier of $\uparrow P=$ the carrier of $P$,

(ii) the internal relation of $\uparrow P=(\text { the internal relation of } P)^{\smile}$, and

(iii) the alternative relation of $\uparrow P=(\text { the alternative relation of } P)^{\mathrm{c}}$.

Let $P$ be a non empty pcs structure. One can check that $\uparrow P$ is non empty.

Next we state three propositions:

(33) Let $P$ be a pcs structure, $p, q$ be elements of $P$, and $p^{\prime}, q^{\prime}$ be elements of $\uparrow P$. If $p=p^{\prime}$ and $q=q^{\prime}$, then $p \leq q$ iff $q^{\prime} \leq p^{\prime}$.

(34) Let $P$ be a pcs structure, $p, q$ be elements of $P$, and $p^{\prime}, q^{\prime}$ be elements of $\uparrow P$. If $p=p^{\prime}$ and $q=q^{\prime}$, then if $p \sim q$, then $p^{\prime} \not q^{\prime}$.

(35) Let $P$ be a non empty pcs structure, $p, q$ be elements of $P$, and $p^{\prime}, q^{\prime}$ be elements of $\uparrow P$. If $p=p^{\prime}$ and $q=q^{\prime}$, then if $p^{\prime} \nsim q^{\prime}$, then $p \sim q$.

Let $P$ be a reflexive pcs structure. One can check that $\uparrow P$ is reflexive.

Let $P$ be a transitive pcs structure. Observe that $\uparrow P$ is transitive.

Let $P$ be a $\beta$-reflexive pcs structure. One can verify that $\uparrow P$ is $\beta$-irreflexive.

Let $P$ be a $\beta$-irreflexive pcs structure. One can check that $\uparrow P$ is $\beta$-reflexive.

Let $P$ be a $\beta$-symmetric pcs structure. One can verify that $\uparrow P$ is $\beta$ symmetric.

Let $P$ be a compatible pcs structure. Note that $\uparrow P$ is compatible.

Let $P, Q$ be pcs structures. The functor $P \otimes Q$ yielding a pcs structure is defined by the condition (Def. 41). 
(Def. 41) $P \otimes Q=\langle$ : the carrier of $P$, the carrier of $Q:$, (the internal relation of $P) \times($ the internal relation of $Q)$, [^the alternative relation of $P$, the alternative relation of $\left.\left.Q^{\wedge}\right]\right\rangle$.

Let $P, Q$ be pcs structures. One can check that $P \otimes Q$ is strict.

Let $P, Q$ be non empty pcs structures. Note that $P \otimes Q$ is non empty.

One can prove the following propositions:

(36) Let $P, Q$ be pcs structures, $p, q$ be elements of $P \otimes Q, p_{1}, p_{2}$ be elements of $P$, and $q_{1}, q_{2}$ be elements of $Q$. If $p=\left\langle p_{1}, q_{1}\right\rangle$ and $q=\left\langle p_{2}, q_{2}\right\rangle$, then $p \leq q$ iff $p_{1} \leq p_{2}$ and $q_{1} \leq q_{2}$.

(37) Let $P, Q$ be pcs structures, $p, q$ be elements of $P \otimes Q, p_{1}, p_{2}$ be elements of $P$, and $q_{1}, q_{2}$ be elements of $Q$. If $p=\left\langle p_{1}, q_{1}\right\rangle$ and $q=\left\langle p_{2}, q_{2}\right\rangle$, then if $p \sim q$, then $p_{1} \sim p_{2}$ or $q_{1} \sim q_{2}$.

(38) Let $P, Q$ be non empty pcs structures, $p, q$ be elements of $P \otimes Q, p_{1}, p_{2}$ be elements of $P$, and $q_{1}, q_{2}$ be elements of $Q$. If $p=\left\langle p_{1}, q_{1}\right\rangle$ and $q=\left\langle p_{2}\right.$, $\left.q_{2}\right\rangle$, then if $p_{1} \sim p_{2}$ or $q_{1} \sim q_{2}$, then $p \sim q$.

Let $P, Q$ be reflexive pcs structures. Observe that $P \otimes Q$ is reflexive.

Let $P, Q$ be transitive pcs structures. One can check that $P \otimes Q$ is transitive.

Let $P$ be a pcs structure and let $Q$ be a $\beta$-reflexive pcs structure. One can check that $P \otimes Q$ is $\beta$-reflexive.

Let $P$ be a $\beta$-reflexive pcs structure and let $Q$ be a pcs structure. One can check that $P \otimes Q$ is $\beta$-reflexive.

Let $P, Q$ be $\beta$-symmetric pcs structures. One can verify that $P \otimes Q$ is $\beta$-symmetric.

Let $P, Q$ be compatible pcs structures. Observe that $P \otimes Q$ is compatible.

Let $P, Q$ be pcs structures. The functor $P \longmapsto Q$ yielding a pcs structure is defined as follows:

(Def. 42) $P \longmapsto Q=\uparrow P \otimes Q$.

Let $P, Q$ be pcs structures. One can check that $P \longmapsto Q$ is strict.

Let $P, Q$ be non empty pcs structures. Note that $P \longmapsto Q$ is non empty.

Next we state three propositions:

(39) Let $P, Q$ be pcs structures, $p, q$ be elements of $P \longmapsto Q, p_{1}, p_{2}$ be elements of $P$, and $q_{1}, q_{2}$ be elements of $Q$. If $p=\left\langle p_{1}, q_{1}\right\rangle$ and $q=\left\langle p_{2}\right.$, $\left.q_{2}\right\rangle$, then $p \leq q$ iff $p_{2} \leq p_{1}$ and $q_{1} \leq q_{2}$.

(40) Let $P, Q$ be pcs structures, $p, q$ be elements of $P \longmapsto Q, p_{1}, p_{2}$ be elements of $P$, and $q_{1}, q_{2}$ be elements of $Q$. If $p=\left\langle p_{1}, q_{1}\right\rangle$ and $q=\left\langle p_{2}\right.$, $\left.q_{2}\right\rangle$, then if $p \sim q$, then $p_{1} \nsim p_{2}$ or $q_{1} \sim q_{2}$.

(41) Let $P, Q$ be non empty pcs structures, $p, q$ be elements of $P \longmapsto Q$, $p_{1}, p_{2}$ be elements of $P$, and $q_{1}, q_{2}$ be elements of $Q$. If $p=\left\langle p_{1}, q_{1}\right\rangle$ and $q=\left\langle p_{2}, q_{2}\right\rangle$, then if $p_{1} \nsim p_{2}$ or $q_{1} \sim q_{2}$, then $p \sim q$.

Let $P, Q$ be reflexive pcs structures. One can check that $P \longmapsto Q$ is reflexive. 
Let $P, Q$ be transitive pcs structures. Observe that $P \longmapsto Q$ is transitive.

Let $P$ be a pcs structure and let $Q$ be a $\beta$-reflexive pcs structure. Note that $P \longmapsto Q$ is $\beta$-reflexive.

Let $P$ be a $\beta$-irreflexive pcs structure and let $Q$ be a pcs structure. One can verify that $P \longmapsto Q$ is $\beta$-reflexive.

Let $P, Q$ be $\beta$-symmetric pcs structures. Note that $P \longmapsto Q$ is $\beta$-symmetric.

Let $P, Q$ be compatible pcs structures. Note that $P \longmapsto Q$ is compatible.

Let $P, Q$ be pcs's. Note that $P \longmapsto Q$ is pcs-like.

Let $P$ be a pcs structure and let $S$ be a subset of $P$. We say that $S$ is self-coherent if and only if:

(Def. 43) For all elements $x, y$ of $P$ such that $x \in S$ and $y \in S$ holds $x \sim y$.

Let $P$ be a pcs structure. Observe that every subset of $P$ which is empty is also self-coherent.

Let $P$ be a pcs structure. One can check that there exists a subset of $P$ which is empty.

Let $P$ be a pcs structure and let $F$ be a family of subsets of $P$. We say that $F$ is self-coherent-membered if and only if:

(Def. 44) For every subset $S$ of $P$ such that $S \in F$ holds $S$ is self-coherent.

Let $P$ be a pcs structure. Observe that there exists a family of subsets of $P$ which is non empty and self-coherent-membered.

Let $P$ be a pcs structure and let $D$ be a set. The functor $\mathcal{P}_{\mathrm{IR}}(P, D)$ yields a binary relation on $D$ and is defined by the condition (Def. 45).

(Def. 45) Let $A, B$ be sets. Then $\langle A, B\rangle \in \mathcal{P}_{\mathrm{IR}}(P, D)$ if and only if the following conditions are satisfied:

(i) $A \in D$,

(ii) $B \in D$, and

(iii) for every set $a$ such that $a \in A$ there exists a set $b$ such that $b \in B$ and $\langle a, b\rangle \in$ the internal relation of $P$.

The functor $\mathcal{P}_{\mathrm{TR}}(P, D)$ yielding a binary relation on $D$ is defined by the condition (Def. 46).

(Def. 46) Let $A, B$ be sets. Then $\langle A, B\rangle \in \mathcal{P}_{\mathrm{TR}}(P, D)$ if and only if the following conditions are satisfied:

(i) $A \in D$,

(ii) $B \in D$, and

(iii) for all sets $a, b$ such that $a \in A$ and $b \in B$ holds $\langle a, b\rangle \in$ the alternative relation of $P$.

Next we state two propositions:

(42) Let $P$ be a pcs structure, $D$ be a family of subsets of $P$, and $A, B$ be sets. Then $\langle A, B\rangle \in \mathcal{P}_{\mathrm{IR}}(P, D)$ if and only if the following conditions are satisfied: 
(i) $A \in D$,

(ii) $B \in D$, and

(iii) for every element $a$ of $P$ such that $a \in A$ there exists an element $b$ of $P$ such that $b \in B$ and $a \leq b$.

(43) Let $P$ be a pcs structure, $D$ be a family of subsets of $P$, and $A, B$ be sets. Then $\langle A, B\rangle \in \mathcal{P}_{\mathrm{TR}}(P, D)$ if and only if the following conditions are satisfied:

(i) $A \in D$,

(ii) $B \in D$, and

(iii) for all elements $a, b$ of $P$ such that $a \in A$ and $b \in B$ holds $a \sim b$.

Let $P$ be a pcs structure and let $D$ be a set. The functor $\mathcal{P}(P, D)$ yielding a pcs structure is defined by:

(Def. 47) $\mathcal{P}(P, D)=\left\langle D, \mathcal{P}_{\mathrm{IR}}(P, D), \mathcal{P}_{\mathrm{TR}}(P, D)\right\rangle$.

Let $P$ be a pcs structure and let $D$ be a family of subsets of $P$. We introduce $\mathcal{P}(D)$ as a synonym of $\mathcal{P}(P, D)$.

Let $P$ be a pcs structure and let $D$ be a non empty set. Observe that $\mathcal{P}(P, D)$ is non empty.

Next we state four propositions:

(44) Let $P$ be a pcs structure, $D$ be a set, and $p, q$ be elements of $\mathcal{P}(P, D)$. Suppose $p \leq q$. Let $p^{\prime}$ be an element of $P$. If $p^{\prime} \in p$, then there exists an element $q^{\prime}$ of $P$ such that $q^{\prime} \in q$ and $p^{\prime} \leq q^{\prime}$.

(45) Let $P$ be a pcs structure, $D$ be a non empty family of subsets of $P$, and $p, q$ be elements of $\mathcal{P}(D)$. Suppose that for every element $p^{\prime}$ of $P$ such that $p^{\prime} \in p$ there exists an element $q^{\prime}$ of $P$ such that $q^{\prime} \in q$ and $p^{\prime} \leq q^{\prime}$. Then $p \leq q$.

(46) Let $P$ be a pcs structure, $D$ be a set, and $p, q$ be elements of $\mathcal{P}(P, D)$. Suppose $p \sim q$. Let $p^{\prime}, q^{\prime}$ be elements of $P$. If $p^{\prime} \in p$ and $q^{\prime} \in q$, then $p^{\prime} \sim q^{\prime}$.

(47) Let $P$ be a pcs structure, $D$ be a non empty family of subsets of $P$, and $p, q$ be elements of $\mathcal{P}(D)$. Suppose that for all elements $p^{\prime}, q^{\prime}$ of $P$ such that $p^{\prime} \in p$ and $q^{\prime} \in q$ holds $p^{\prime} \sim q^{\prime}$. Then $p \sim q$.

Let $P$ be a pcs structure and let $D$ be a set. One can check that $\mathcal{P}(P, D)$ is strict.

Let $P$ be a reflexive pcs structure and let $D$ be a family of subsets of $P$. Note that $\mathcal{P}(D)$ is reflexive.

Let $P$ be a transitive pcs structure and let $D$ be a set. One can check that $\mathcal{P}(P, D)$ is transitive.

Let $P$ be a $\beta$-reflexive pcs structure and let $D$ be a self-coherent-membered family of subsets of $P$. One can check that $\mathcal{P}(D)$ is $\beta$-reflexive. 
Let $P$ be a $\beta$-symmetric pcs structure and let $D$ be a family of subsets of $P$. Observe that $\mathcal{P}(D)$ is $\beta$-symmetric.

Let $P$ be a compatible pcs structure and let $D$ be a family of subsets of $P$. Note that $\mathcal{P}(D)$ is compatible.

Let $P$ be a pcs structure. The functor pcs-coherent-power $P$ yields a set and is defined as follows:

(Def. 48) pcs-coherent-power $P=\{X ; X$ ranges over subsets of $P: X$ is selfcoherent\}.

We now state the proposition

(48) For every pcs structure $P$ and for every set $X$ such that $X \in$ pcs-coherent-power $P$ holds $X$ is a self-coherent subset of $P$.

Let $P$ be a pcs structure. Note that pcs-coherent-power $P$ is non empty.

Let $P$ be a pcs structure. Then pcs-coherent-power $P$ is a family of subsets of $P$.

Let $P$ be a pcs structure. Observe that pcs-coherent-power $P$ is self-coherentmembered.

Let $P$ be a pcs structure. The functor $\mathcal{P}(P)$ yielding a pcs structure is defined by:

(Def. 49) $\quad \mathcal{P}(P)=\mathcal{P}$ (pcs-coherent-power $P$ ).

Let $P$ be a pcs structure. Note that $\mathcal{P}(P)$ is strict.

Let $P$ be a pcs structure. Note that $\mathcal{P}(P)$ is non empty.

Let $P$ be a reflexive pcs structure. One can verify that $\mathcal{P}(P)$ is reflexive.

Let $P$ be a transitive pcs structure. One can check that $\mathcal{P}(P)$ is transitive.

Let $P$ be a $\beta$-reflexive pcs structure. Note that $\mathcal{P}(P)$ is $\beta$-reflexive.

Let $P$ be a $\beta$-symmetric pcs structure. Note that $\mathcal{P}(P)$ is $\beta$-symmetric.

Let $P$ be a compatible pcs structure. Note that $\mathcal{P}(P)$ is compatible.

Let $P$ be a pcs. Observe that $\mathcal{P}(P)$ is pcs-like.

\section{REFERENCES}

[1] Grzegorz Bancerek. König's theorem. Formalized Mathematics, 1(3):589-593, 1990.

[2] Grzegorz Bancerek. The "way-below" relation. Formalized Mathematics, 6(1):169-176, 1997.

[3] Chantal Berline and Klaus Grue. A kappa-denotational semantics for map theory in ZFC + SI. Theoretical Computer Science, 179(1-2):137-202, 1997.

[4] Czesław Byliński. Functions and their basic properties. Formalized Mathematics, 1(1):5565, 1990.

[5] Czesław Byliński. The modification of a function by a function and the iteration of the composition of a function. Formalized Mathematics, 1(3):521-527, 1990.

[6] Czesław Byliński. Partial functions. Formalized Mathematics, 1(2):357-367, 1990.

[7] Czesław Byliński. Some basic properties of sets. Formalized Mathematics, 1(1):47-53, 1990.

[8] Adam Grabowski and Robert Milewski. Boolean posets, posets under inclusion and products of relational structures. Formalized Mathematics, 6(1):117-121, 1997.

[9] Zbigniew Karno. Separated and weakly separated subspaces of topological spaces. Formalized Mathematics, 2(5):665-674, 1991. 
[10] Artur Korniłowicz. Cartesian products of relations and relational structures. Formalized Mathematics, 6(1):145-152, 1997.

[11] J.L. Krivine. Lambda-calculus, types and models. Ellis \& Horwood, 1993.

[12] Beata Madras. Product of family of universal algebras. Formalized Mathematics, 4(1):103-108, 1993.

[13] Markus Moschner. Basic notions and properties of orthoposets. Formalized Mathematics, 11(2):201-210, 2003.

[14] Konrad Raczkowski and Paweł Sadowski. Equivalence relations and classes of abstraction. Formalized Mathematics, 1(3):441-444, 1990.

[15] Andrzej Trybulec. Domains and their Cartesian products. Formalized Mathematics, 1(1):115-122, 1990.

[16] Andrzej Trybulec. Tarski Grothendieck set theory. Formalized Mathematics, 1(1):9-11, 1990.

[17] Andrzej Trybulec. Tuples, projections and Cartesian products. Formalized Mathematics, 1(1):97-105, 1990.

[18] Andrzej Trybulec. Many-sorted sets. Formalized Mathematics, 4(1):15-22, 1993.

[19] Wojciech A. Trybulec and Grzegorz Bancerek. Kuratowski - Zorn lemma. Formalized Mathematics, 1(2):387-393, 1990.

[20] Zinaida Trybulec. Properties of subsets. Formalized Mathematics, 1(1):67-71, 1990.

[21] Edmund Woronowicz. Relations and their basic properties. Formalized Mathematics, 1(1):73-83, 1990.

[22] Edmund Woronowicz. Relations defined on sets. Formalized Mathematics, 1(1):181-186, 1990.

[23] Edmund Woronowicz and Anna Zalewska. Properties of binary relations. Formalized Mathematics, 1(1):85-89, 1990.

Received August 28, 2007 